\title{
Adaptive FIR Filtering of Range Sidelobes for Air and Spaceborne Rain Mapping
}

\author{
Stephen P. Lohmeier \\ University of Kansas, \\ Radar Systems and Remote Sensing Laboratory, \\ Information Technology and Telecommunications Center
}

\begin{abstract}
This paper describes an adaptive finite-impulse response (FIR) filtering technique to reduce the range sidelobes due to surface return inherent in a pulse-compression radar used for air or spaceborne rain mapping. Its sidelobe suppression performance exceeds traditional windowing methods without a reduction in range resolution.
\end{abstract}

\section{INTRODUCTION}

Satellite measurements of rainfall rate are currently performed using both active and passive microwave sensors (i.e., radars and radiometers). The Tropical Rainfall Measuring Mission (TRMM) satellite, for example, caries both types of sensors in its sensor suite-the TRMM Microwave Imager (TMI), a passive sensor, and the Precipitation Radar (PR), an active sensor. The main advantages of a passive microwave approach over an active one are the simplicity and cost of the sensor. However, passive sensor techniques for measuring rain do not work over land. In this regard, radar appears to be more attractive for measuring rainfall rate from space. Further, one of the major disadvantages of space-based radars is the high peak power required to operate a pulse-modulated radar in a low PRF, low duty-cycle mode. To achieve the required power level either a microwave tube transmitter or multiple microwave power amplifiers operating in parallel are required.

Pulse compression using a linear FM waveform (LFM) is a well-known technique that can significantly reduce the required transmit power. Pulse compression relies on illuminating the target over a finite band of frequencies at low power for a long duration. Subsequent signal processing results in a range profile of the target. Because of the finite band of frequencies involved, range sidelobes appear in the range profile. In measuring rainfall rate using pulse compression, the range sidelobes associated with the return from the air-earth interface can be stronger than the return from the rain itself, making measurement of the rain impossible. However, with proper range-sidelobe suppression the effects of range sidelobes can be reduced to an acceptable level. Past efforts have focused primarily on windowing techniques that have achieved up to $-55 \mathrm{~dB}$ [1] sidelobe levels. Although others have used wavelets to achieve suppression [2]. To measure light rain, however, range sidelobes must be down more than $-60 \mathrm{~dB}$ from the main return [3].

In this paper a novel concept using adaptive FIR filtering effectively reduces the range sidelobes to an acceptable level. The techniques of adaptive cancellation and filtering are well known, well developed, and have been applied in a variety of applications. Most adaptive algorithms rely on knowledge of the desired signal to cancel unknown interferers. However, in this application, some information about the interferer (or the surface return) is known, but the desired signals (or rainfall returns) are unknown. Here an algorithm that computes the filter weights under these circumstances is presented. Simulation results using the algorithm are presented using idealized data.

Section II describes the nature of the problem, Section III describes the solution, Section IV describes the results obtained, and Section V concludes the paper.

\section{THE NATURE OF THE PROBLEM}

In a traditional short-pulse radar approach the range resolution is determined by the pulse width of the transmitted pulse and there are no range sidelobe effects. In contrast, an LFM radar will have a range resolution inversely proportional to the bandwidth of the chirp and range sidelobes corresponding to a sinc function that are a minimum of $13 \mathrm{~dB}$ down from the return at the target range. This is illustrated in Figure 1. In this figure the solid line depicts the return received by a short pulse radar. The surface return at around $2000 \mathrm{~m}$ is $60 \mathrm{~dB}$ higher than the rain return at other ranges. The dashed return shown shows the simulated return received by a pulse compression radar with rectangular (or no) windowing. As can be seen in the figure, the rain return is masked by the range sidelobes of the surface return. Figure 2 , shows the situation where windowing is applied. In this figure the solid line depicts the return received by a short pulse radar, and the dashed line shows the return received by a pulse-compression radar where the chirp has been weighted by a window function. The first sidelobe is reduced by $45 \mathrm{~dB}$ from the main lobe, but the range resolution is substantially degraded by the windowing process. While windowing is a viable technique for range sidelobe suppression it suffers from a reduction in bandwidth. The FIR method proposed does not suffer from this limitation. 


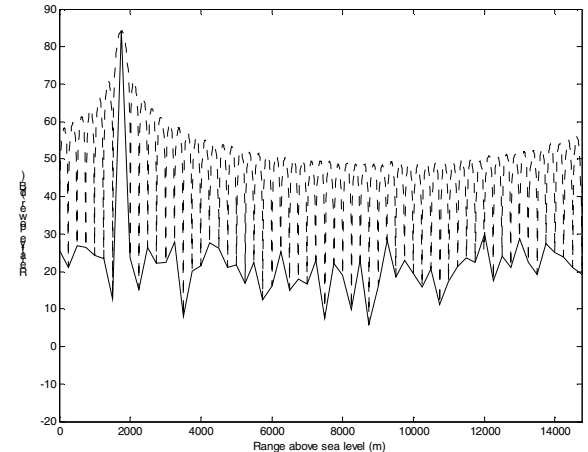

Fig. 1. Comparison of short pulse radar and LFM radar returns. The solid line depicts the return received by a short pulse radar. The surface return at around $2000 \mathrm{~m}$ is $60 \mathrm{~dB}$ higher than the rain return at other ranges. The dotted line depicts the simulated return received by a pulse compression radar with rectangular (or no) windowing. The rain return is masked by the range sidelobes of the surface return.

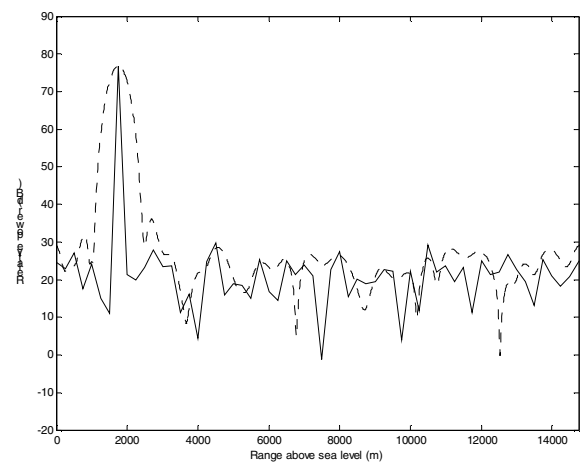

Fig. 2. The solid line depicts the return received by a short pulse radar, and the dotted line shows the return received by a pulse-compression radar where the chirp has been weighted by a window function. The first sidelobe is reduced by $45 \mathrm{~dB}$ from the main lobe, but the range resolution is substantially degraded by the windowing process.

\section{THE SOLUTION}

In LFM processing, the return in the time (or range) domain is determined by computing an inverse Fourier transform (IFT) of the frequency response over the bandwidth of the chirp. When windowing is used, the frequency response is multiplied by the window function prior to performing the IFT. Mathematically this is equivalent to performing the IFT of the unwindowed frequency response and convolving it with the IFT of the window function.

In the FIR approach, the frequency response is convolved with the frequency response of the filter prior to performing the IFT. Mathematically this equivalent to performing the IFT of the frequency response (or time domain response) and multiplying it by IFT the filter frequency response (or impulse response). The impulse response of the filter in this case is effectively a notch in the time domain at the range of the surface return, but it is not implemented directly in this manner.
Figure 3 shows a block diagram depicting the overall approach. The FIR filter coefficients are computed using a Wiener filter for each frequency sweep in which the surface return is expected to be different. Rather than actually filtering out the surface return the FIR filter removes the rain return from the composite return and then subtracts the surface-only return from the composite return. This is done because it is easier to isolate the surface return than the rain return as it may be masked by the surface return sidelobes.

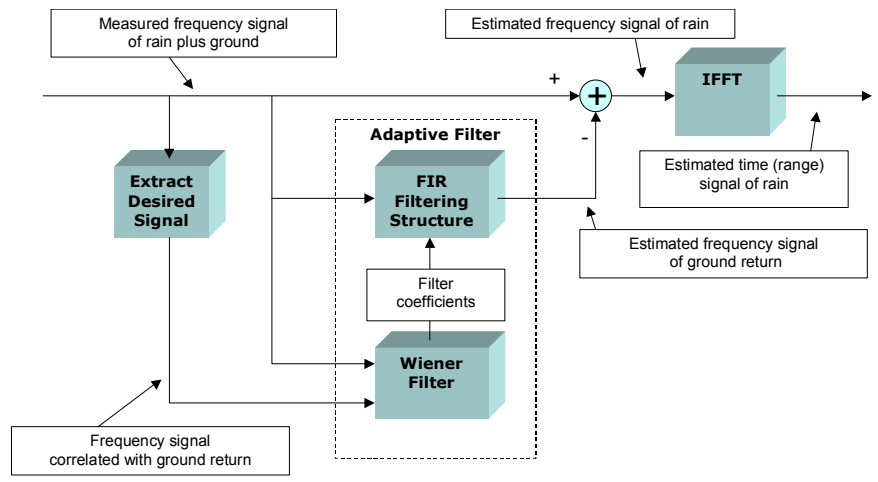

Fig. 3. Adaptive FIR filter block diagram.

The FIR filter is implemented as a convolution in the frequency domain in a tapped delay line (or direct form) architecture as shown in Figure 4. Convolution is more efficient than performing an IFT and multiplying in this case as the number of filter taps is much smaller than the number of frequency samples.

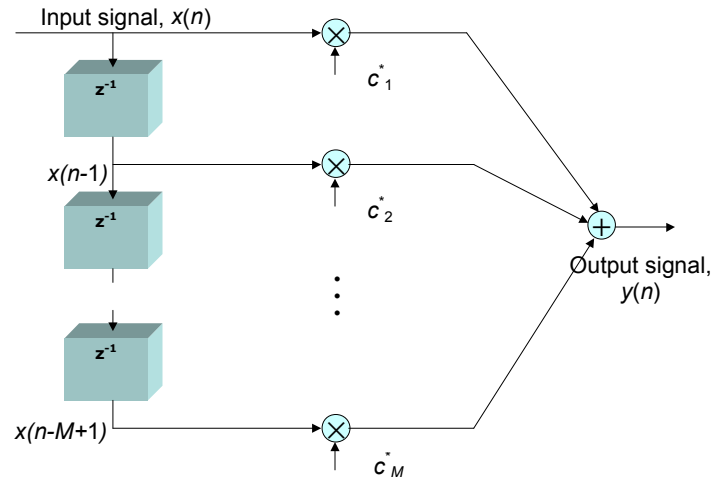

Fig. 4. Direct-form FIR filter structure.

In vector form we can solve for the filter coefficients, $\mathbf{c}$, using the Wiener filter equations

$$
\begin{aligned}
& \mathbf{c}=\hat{\mathbf{R}}^{-1} \hat{\mathbf{d}} \\
& \hat{\mathbf{R}}=\frac{1}{N} \sum_{n=0}^{N-1} \mathbf{x} \mathbf{x}^{H} \\
& \hat{\mathbf{d}}=\frac{1}{N} \sum_{n=0}^{N-1} \mathbf{x} \mathbf{y}^{H}
\end{aligned}
$$




$\begin{array}{ll}\begin{array}{l}\text { where } \\ M\end{array} & \begin{array}{l}\text { is the number of time taps (or filter } \\ \text { coefficients) }\end{array} \\ N & \begin{array}{l}\text { is the number of frequency samples } \\ \text { is the tap index }\end{array} \\ n & \begin{array}{l}\text { is the sample index } \\ \text { is an } \mathrm{M} \times \mathrm{N} \text { circulant matrix of actual } \\ \text { signals (frequency samples of composite } \\ \text { return in rows and shifted versions in } \\ \text { columns) }\end{array} \\ \begin{array}{l}\text { is a } 1 \mathrm{~N} \text { vector of desired signals } \\ \text { (frequency samples of ground return) }\end{array} \\ \begin{array}{l}\text { is the autocovariance matrix of the actual } \\ \text { signal } \\ \text { is the covariance matrix of the actual signal } \\ \text { with the desired signal }\end{array} \\ \begin{array}{l}\text { is a vector of filter coefficients } \\ c(m, m) 1)\end{array}\end{array}$

The desired signal extraction, which in this case is the frequency domain representation of the ground return is performed by performing an IFT on the composite frequencydomain return, isolating the return from the surface, and then performing a forward Fourier transform (FT) to get the surface return in the frequency domain. The surface return is isolated as follows:

1. Locate the range bin containing the maximum return power - this is the first estimate of the ground location

2. Verify that the range is reasonable for ground return based on previous estimates of range to ground and other metrics

3. Include adjacent range bins if their power levels are within $13 \mathrm{~dB}$ of the maximum - range sidelobes would mask lower returns

4. Zero out returns at other ranges

\section{RESULTS}

The filter was applied to simulated data assuming that surface and rain magnitude returns are Rayleigh distributed (i.e. bivariate Gaussian I and Q signals), and returns from adjacent range bins are independent and uncorrelated. The second assumption is represents the worst case because in most rain storms the rain returns are correlated to neighboring range bins. The radar system parameters assumed were:

- Range Resolution $250 \mathrm{~m}$

- Number of Range Bins

60

- Range Window

$15 \mathrm{~km}$

- Frequency Resolution

$10 \mathrm{kHz}$

- Number of Frequency Samples

60

- Bandwidth

- Number of Samples Averaged

$600 \mathrm{kHz}$

64

$1750 \mathrm{~m}$

$-60 \mathrm{~dB}$

- Rain to Ground Ratio

- Number of FIR Filter Taps
The system parameters bear resemblance to those of the TRMM Precipitation Radar, but the data is simulated. Figure 5 shows the results after application of the filter. As can be seen in the lower right plot the filter completely removes the effects of the surface return range sidelobes leaving only the rain return.
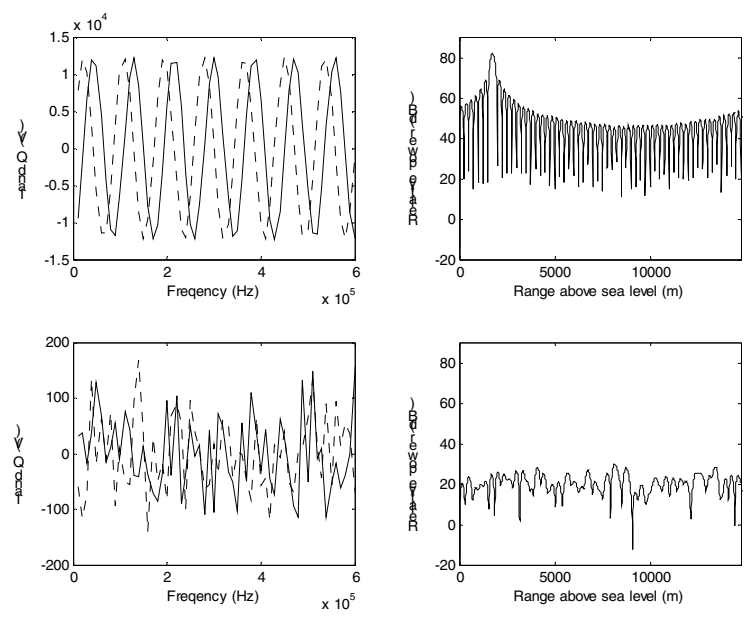

Fig. 5. Unfiltered vs. filtered return. The upper row of plots shows the unfiltered frequency and time (or range) domain returns of the composite return. Range sidelobes clearly mask the rain return. The second row of plots shows the rain-only return after filtering. The rain return is now clearly visible after removing the surface return and surface range sidelobes.

\section{CONCLUSIONS}

This paper describes an adaptive FIR filter that can be used instead of windowing to remove range sidelobes from surface returns in air and spaceborne rain mapping radars using LFM pulse compression. Future plans include applying this technique to TRMM data, and exploring other filtering paradigms.

\section{REFERENCES}

[1] A Tanner, S. L. Durden, R. Denning, E. Im, F. K. Li, W. Ricketts, and W. Wilson, "Pulse compression with very low sidelobes in an airborne rain mapping radar," IEEE Trans. Geosci. Remote Sensing, vol. 32, pp. 211-213, Jan. 1994.

[2] N. Ehara, I. Sasase, and S. Mori, "Range sidelobe suppression by locally weighting filter," Electronics and Communications in Japan, part I, vol. 79, no. 1, pp. 6473, 1996. [Translated from Denshi Joho Tsushin Gakkai Ronbunshi, vol. 78-B-II, no. 1, pp.21-29, Jan. 1995].

[3] T. Ihara and K. Nakamura, "A feasibility study of rain radar for the tropical rainfall measuring mission: 4. A discussion of pulse compression and adaptive scanning," J. Comm. Res. Lab., vol. 35, no. 145, pp. 149-161, 1988. 